FORMATION Formation emploi

Revue française de sciences sociales

131 | Juillet-Septembre 2015

Le Bac Pro a 30 ans

\title{
Des bacheliers professionnels sur le pont des Arts, du rêve à la réalité ?
}

Vocational baccalauréat holders holding onto their dreams on the pont des

Arts?

Berufsabiturienten auf dem Kunstweg, Traum oder Wirklichkeit?

Bachilleres técnicos sobre el puente de las Artes, ¿del sueño a la realidad?

Magali Danner et Christine Guégnard

\section{OpenEdition}

Journals

Édition électronique

URL : http://journals.openedition.org/formationemploi/4505

DOI : 10.4000/formationemploi.4505

ISSN : 2107-0946

Éditeur

La Documentation française

Édition imprimée

Date de publication : 15 octobre 2015

Pagination : 141-162

ISSN : 0759-6340

Référence électronique

Magali Danner et Christine Guégnard, « Des bacheliers professionnels sur le pont des Arts, du rêve à la réalité ? », Formation emploi [En ligne], 131 | Juillet-Septembre 2015, mis en ligne le 15 octobre 2017, consulté le 30 octobre 2020. URL : http://journals.openedition.org/formationemploi/4505 ; DOI : https://doi.org/10.4000/formationemploi.4505

(c) Tous droits réservés 


\title{
Des bacheliers professionnels sur le pont des Arts, du rêve à la réalité ?
}

\author{
Magali DanNeR \\ Enseignante-chercheure en sciences de l'éducation à l'Institut de recherche sur l'éducation, \\ université Bourgogne Franche-Comté \\ Christine GuÉGNARD \\ Chargée d'études à l'Institut de recherche sur l'éducation, université Bourgogne Franche-Comté, \\ pour le centre associé au Céreq de Dijon, sciences de l'éducation
}

Résumé

Des bacheliers professionnels sur le pont des Arts, du rêve à la réalité ?

Face au champ des possibles des formations, les classes préparatoires aux écoles supérieures d'art constituent un choix original pour un jeune, encore plus pour un bachelier professionnel. Élèves atypiques dans des classes atypiques, leur présence interpelle : pour quelles raisons choisissent-ils ces études, quels sont leurs objectifs et qu'attendent-ils de l'année préparatoire ? Les déterminants de l'orientation de ces bacheliers professionnels, invisibles au sens statistique, sont abordés à partir des représentations des jeunes.

Mots-clés : enseignement art-architecture, CPGE - classe préparatoire, baccalauréat professionnel, politique de l'éducation, poursuite d'études, accès a l'enseignement supérieur, orientation scolaire-professionnelle, sélection

Abstract

Vocational baccalauréat holders holding onto their dreams on the pont des Arts?

In the context of the scope of the possibilities of higher studies, prep art schools are a novel choice for youths, even more so for those with a vocational baccalauréat. Atypical students in atypical classes, their presence begs the questions: why do they choose these studies, what are their goals and what they do expect of this preparatory year? The determinants of the choices of these youths, who are invisible in the statistical sense, are discussed from the point of view of their representations.

Keywords: art \& architecture education, preparatory class for entrance into elite schools, vocational baccalaureate, education policy, continuation of education, access to higher education, school guidance $\&$ vocational guidance, selection

Journal of Economic Literature: I 21

Traduction : Auteures. 
La mise en place, en décembre 2014, d'une mission de réflexion sur l'ouverture d'une nouvelle filière post-baccalauréat pour les bacheliers professionnels est l'opportunité de s'interroger sur la place de ces jeunes dans l'offre globale des formations supérieures.

La création du baccalauréat professionnel, en 1985, répondait à un double objectif : favoriser l'entrée immédiate dans la vie active et mener $80 \%$ d'une classe d'âge au niveau du baccalauréat en $2000 \ldots$ mais après ? (Beaud, 2002). Ce diplôme a connu un développement rapide, accentué par les dernières réformes de l'enseignement professionnel. Une récente publication du ministère de l'Éducation nationale, de l'Enseignement supérieur et de la Recherche (2015) constate que 31 \% des bacheliers sont désormais diplômés d'un baccalauréat professionnel. Par ricochet, la part des bacheliers professionnels qui entreprennent des études supérieures l'année suivant leur diplôme progresse depuis treize ans et s'établit à $33 \%$ en 2013 hors alternance (17\% en 2000). Plus de $80 \%$ d'entre eux souhaitaient poursuivre des études en sections de techniciens supérieurs (STS), où ils sont accueillis de droit dès lors qu'ils ont une mention bien ou très bien ${ }^{1}$. Dans les faits, cependant, seuls deux sur cinq y sont effectivement admis.

L'orientation vers l'université peut devenir alors un choix par défaut (Felouzis, 2001) "teinté de déception» (Paivandi, 2011), voire "une impasse» (Beaud, Pialoux, 2001). Cette alternative est d'autant plus mal vécue que la probabilité d'être diplômé est faible. De fait, $15 \%$ des bacheliers professionnels sont toujours en licence trois ans après leur première inscription, alors que le taux de réussite en BTS (brevet de technicien supérieur) est de $50 \%$, toutes spécialités confondues (MENESR, 2015).

Quant à leur présence en classes préparatoires publiques aux grandes écoles (CPGE), elle reste anecdotique. Ceci est notamment le cas pour les classes préparatoires publiques aux écoles supérieures d'art. Celles-ci, avec moins de 500 élèves inscrits, sont d'ailleurs elles-mêmes absentes des tableaux statistiques, se situant dans l'ombre des CPGE qui regroupent 34700 étudiants en première année à la rentrée 2013.

Trop spécifiques, les classes préparatoires publiques aux écoles supérieures d'art font aussi rarement l'objet de recherche scientifique. N'étant pas inscrites dans le système LMD (licence-master-doctorat), elles ne sont pas référencées dans la procédure actuelle d'orientation post-bac via Internet (interface Admission Post-Bac), n'offrent pas le double statut d'élèves et d'étudiants et n'ouvrent pas droit au régime étudiant de la sécurité sociale ni aux bourses sur critères sociaux ${ }^{2}$.

La classe préparatoire aux écoles supérieures d'art se distingue de la plupart des CPGE par une spécialisation des compétences et un répertoire de formations supérieures ou de métiers clairement ciblés. Centrée sur la pratique plutôt que sur l'enseignement magis-

1. Décret $\mathrm{N}^{\circ} 2007-540$, du 11 avril 2007, Journal Officiel du 12/04/2007.

2. Même si quelques rares établissements ont obtenu un agrément permettant à leurs élèves de bénéficier de la sécurité sociale étudiante, et de manière exceptionnelle, du statut d'étudiant et de bourse. 
tral, elle s'inscrit dans la continuité d'un contexte de scolarisation propre à l'enseignement secondaire professionnel qui a pu intéresser ces jeunes. Cet aspect engageant et l'existence d'une sélection à l'entrée rendent peu probable une orientation par défaut des bacheliers professionnels inscrits. Toutefois, pour les bacheliers professionnels qui auraient préféré être admis en STS, ou même dans une MANAA (Classe de mise à niveau en arts appliqués, intégrée généralement dans un lycée) préparant aux BTS, la classe préparatoire peut devenir une solution de repli. Il est vrai que la classe MANAA paraît a priori mieux adaptée à leur profil scolaire et peut avoir eu leur préférence. Néanmoins, la classe préparatoire autorise aussi une logique d'études professionnalisantes avec la possibilité, une fois admis aux beaux-arts, de valider en trois ans le Diplôme national d'arts et techniques (DNAT). Dès lors, "Le choix du supérieur court dans un domaine artistique apparaît donc comme une volonté d'orientation très marquée, et bien spécifique par rapport à l'ensemble de l'offre de formation" (Sulzer, 2000, p. 20). Le cursus long des écoles supérieures d'art, sanctionné par le Diplôme national supérieur d'expression plastique (DNSEP), peut luimême être interrompu au terme de trois années avec l'obtention du Diplôme national d'arts plastiques (DNAP).

Pour autant, cette hypothèse d'une orientation choisie ne présage pas nécessairement d'un rapport simple aux études entreprises. En effet, cette formation post-baccalauréat s'adresse à une certaine élite puisque, de la classe préparatoire à l'obtention d'un diplôme en école supérieure d'art, la sélection rythme les études. Élitistes, ces cursus le sont aussi, de façon plus subtile, par la prégnance d'une idéologie du don qui domine le champ de la pratique artistique (Liot, 2004) et par la valorisation d'une culture « savante », d'autant plus discriminante que peu d'heures, dans le secondaire, sont dévolues aux enseignements artistiques définis progressivement comme des options.

Les modalités d'orientation vers l'enseignement secondaire professionnel et le curriculum spécifique qui le caractérise préparent mal ces bacheliers à surmonter les étapes de la sélection académique (Fouquet, 2014) et à se projeter dans des activités professionnelles en lien avec la conception, l'innovation artistique et la créativité qui sont les finalités des écoles supérieures d'art.

D’ailleurs, le marché du travail artistique lui-même est réputé sélectif par la médiatisation des "winner-take-all markets " ${ }^{3}$ qui laissent peu de perspectives professionnelles aux diplômés, certes compétents, mais dont le talent n’a pas encore été reconnu (Frank, Cook, 1995 ; Menger, 2002). Les études artistiques ne garantissent pas, en effet, la qualité de l'insertion professionnelle (Prélot, 1992), notamment parce que "l'art n'est ni un métier, ni une activité de loisir, mais un hybride anormal entre les deux" (Freidson, 1994, p. 134). Ni le monde de l'art, ni le public ne choisissent de s'intéresser à un artiste au "simple motif qu'il peut exhiber un diplôme d'école» (Talbot, 1996, p. 32).

3. C’est-à-dire un marché où le vainqueur ramasse tout. 
Mieux définir les motivations qui ont amené ces bacheliers professionnels à s'orienter vers la classe préparatoire et le rapport qu'ils entretiennent à ces études est donc de nature à alimenter la réflexion sociologique sur leurs capacités à s'approprier l'offre de formation dans le supérieur et, à travers ces choix d'études atypiques, à échapper, éventuellement, à un certain destin social auquel les prédispose leur formation secondaire (Dubet, Martucelli, 1996).

Leur origine scolaire et leur choix d'orientation en font des étudiants invisibles dans les statistiques, sauf lorsqu'il s'agit d'expliquer les échecs dans le supérieur, qu'il importe de mettre en lumière compte tenu de leur place dans la trilogie des baccalauréats.

\section{Encadré 1 : Note méthodologique}

La recherche repose sur une enquête menée auprès des inscrits en classe préparatoire aux écoles supérieures d'art. Seize classes préparatoires publiques, soit la quasi-totalité des écoles publiques sur le territoire national, ont participé à cette enquête. 323 jeunes, dont dix-neuf bacheliers professionnels, ont répondu à un questionnaire en mars 2014. L'objectif était de connaître le public, les motivations des jeunes, leurs projets, les intérêts de la formation vis-àvis de leurs objectifs, leurs attentes et leurs appréciations sur les conditions d'études. La passation des questionnaires s'effectuait lors d'une séance en cours collectif avec restitution dans des enveloppes individuelles pour garantir leur anonymat. Le taux de participation des élèves a été de 323 répondants sur 364 inscrits, soit $89 \%$.

Pour pallier la contrainte méthodologique posée par ces bacheliers invisibles qui, en raison de la faiblesse de leurs effectifs, ne permettent pas d'analyses statistiques approfondies, il est nécessaire de multiplier le nombre d'indicateurs révélateurs d'une situation. Ainsi, à titre d'exemple, l'engagement des bacheliers envers l'art a été appréhendé à travers cinq indicateurs : le choix d'une spécialisation artistique dans le cursus secondaire, le temps de la décision pour s'orienter vers des études artistiques, le choix de la classe préparatoire comme premier vœu, le projet scolaire et le projet professionnel. C'est par la convergence ou non de ces indicateurs multiples que des conclusions peuvent ensuite être formulées.

Afin de bénéficier d'un regard complémentaire aux propos des élèves, les responsables, directeurs et directrices des classes préparatoires publiques ont aussi été invité-e-s à s'exprimer sur les finalités, les missions, l'organisation de la formation. En effet, chaque responsable de structure, au-delà d'être le garant du bon fonctionnement de la classe préparatoire, fixe des priorités politiques qui s'imposent avec plus ou moins d'évidence aux élèves et enseignants. Les points de vue des jeunes sur leur formation se trouvent influencés, dans une certaine mesure, par ce discours institutionnel. Les questions ouvertes ont été exploitées selon la méthode classique d'analyse de contenu avec une opération de codage thématique en catégories et sous-catégories. Les propos des jeunes et des responsables sont reproduits « en italique » dans le texte.

Cette recherche s'appuie sur une enquête menée auprès des jeunes inscrits en 2013 en classe préparatoire publique (Danner, Guégnard, Albandea, 2014). Dans une première partie, le profil des élèves sera analysé afin de spécifier ce qui différencie les bacheliers professionnels des autres publics inscrits. La problématique centrale de cette recherche est d'apprécier dans quelle mesure ces éléments de distinction peuvent impacter leurs motivations et leur rapport aux études en classe préparatoire. Elle trouvera des réponses 
dans une seconde partie, centrée sur la capacité des bacheliers professionnels à rendre légitime une orientation à laquelle leur parcours antérieur les prépare peu. Cette approche s'appuie sur les points de vue des jeunes, considérés comme des acteurs en quête de sens dans leurs études, c'est-à-dire cherchant à mettre en cohérence leurs projets, leurs motivations et leurs attentes.

\section{Des étudiants fragilisés dans leurs études}

L'exigence des épreuves sélectives pour entrer en écoles supérieures d'art a motivé un nombre croissant de bacheliers à envisager une poursuite d'études préalable dans des structures préparant à ces concours, assurant ainsi l'essor d'une offre de formation publique et privée hétérogène. Cette recherche porte sur les classes préparatoires publiques ${ }^{4}$ qui accueillent des élèves souhaitant s'engager dans une formation artistique et les accompagnent dans la préparation des concours aux écoles supérieures d'art.

La formation dure moins d'une année, si bien que la compréhension des attentes institutionnelles et la maîtrise des codes implicites du travail intellectuel (Coulon, 1997) doivent être rapidement mises en place pour tirer tous les bénéfices de cet accompagnement et augmenter les rapports de chance aux concours. Cette première partie positionne et distingue les bacheliers professionnels dans le monde des classes préparatoires, car selon leur profil scolaire et origine socioculturelle, ces jeunes peuvent vivre différemment cette expérience et ce choix d'étude.

\subsection{Un profil scolaire composite}

Les jeunes admis en classe préparatoire publique sont plutôt issus des filières générales, avec $48 \%$ de bacheliers littéraires (L), $20 \%$ de bacheliers scientifiques (S) et $16 \%$ de bacheliers titulaires d'un baccalauréat économique et social (ES). Les autres inscrits sont titulaires des baccalauréats technologique (9\%), professionnel (6\%), du brevet de technicien ou des métiers d'art (1\%) (tableau 1). D'après ce portrait composite, toutes les filières du secondaire peuvent préparer à une poursuite d'études en classe préparatoire aux écoles supérieures d'art. Néanmoins, la présence des bacheliers professionnels reste discrète au regard des autres bacheliers, ce qui souligne la dimension atypique de cette orientation. Sur des promotions de quinze à trente-cinq inscrits, ils représentent moins de trois élèves dans huit écoles et aucun dans sept autres. Une seule école, parmi les seize enquêtées, accueille six bacheliers professionnels.

4. Une quinzaine, dont la plupart sont signataires de la charte de qualité mise en place par l'Association nationale des classes préparatoires publiques aux écoles supérieures d'art (APPEA) et validée par le ministère de la Culture et de la Communication. 
Tableau 1. Répartition par série des bacheliers en 2013 (en \%)

\begin{tabular}{|c|c|c|c|}
\hline Type de bac & $\begin{array}{l}\text { Classes préparatoires } \\
\text { aux écoles sup. d'art }\end{array}$ & $\begin{array}{l}\text { Étudiants en 1re } \\
\text { année de CPGE } \\
\text { en France }\end{array}$ & Bacheliers diplômés en France \\
\hline $\mathrm{L}$ & 48 & 70 & 8 \\
\hline$S$ & 20 & 9 & 27 \\
\hline ES & 16 & 15 & 17 \\
\hline Technologique & 9 & 5 & 21 \\
\hline Professionnel & 6 & - & 27 \\
\hline Autre & 1 & 1 & - \\
\hline Total & 100 & 100 & 100 \\
\hline Effectif & 323 & 40894 & 589240 \\
\hline
\end{tabular}

Lecture : La part des bacheliers professionnels en classe préparatoire publiques d'art est de $6 \%$.

CPGE : Classe préparatoire aux grandes écoles. L : littéraire ; S : scientifique. ; ES : économique et social .

Sources : Enquête IREDU, 2014 et DEPP, 2014, pp. 187, 245.

Certains d'entre eux ont suivi une spécialisation dans le secondaire (arts appliqués, métiers de la mode, ébéniste, aménagements paysagers...), plutôt cohérente avec le choix de la classe préparatoire. Pour d'autres, cependant, aux cursus plus éloignés (boulanger-pâtissier, commerce, technicien du bâtiment...), leur admission interroge, à moins de considérer qu'elle se justifie par un dossier personnel artistique et/ou une prestation orale qui ont su sensibiliser le jury d'admission. Enseignées au primaire et dans les loisirs extrascolaires, les activités plastiques font l'objet d'une initiation au travers de laquelle ces bacheliers ont pu se découvrir des talents et une vocation pour l'art. Les modalités de sélection pour l'accès à la classe préparatoire favorisent la prévalence de ces critères sur d'autres, plus académiques.

D'après les quinze responsables d'écoles, le nombre de dossiers de candidature varie d'une trentaine à 250, la moyenne étant de 92 candidatures par école. Face à cette pression de la demande, les modalités d'admission reposent sur de nombreuses situations d'évaluation mais toutes les écoles sélectionnent, au minimum, à partir du dossier de travaux artistiques personnels et de l'entretien de motivation avec un jury, auxquels peuvent s'ajouter des épreuves écrites et/ou plastiques.

La motivation, la créativité ou "l'envie d'expérimenter » des jeunes sont perçues comme des éléments déterminants pouvant justifier l'admission d'un "profil atypique présentant un intérêt dans cette orientation", selon un directeur. Enfin, la diversité des publics trouve aussi des explications dans l'éventail de missions explicitées par les responsables : préparer aux concours et constituer des dossiers, développer des capacités créatives, ouvrir sur le monde de l'art, aider à l'orientation et accueillir un public mixte socialement et culturellement " en s'adressant à des élèves de profils différents ". Ainsi, des directeurs soulignent que "des jeunes issus de la diversité culturelle, des jeunes issus de parcours scolaires différents ont la possibilité d'acquérir et de développer des compétences techniques liées à la pratique artistique ». 
Le profil académique des bacheliers professionnels dénote toutefois par rapport à celui des autres élèves. Proportionnellement, ils sont autant à s'inscrire en classe préparatoire dans l'année du baccalauréat que les autres jeunes. Leur moyenne d'âge pour l'obtention de ce diplôme, 19 ans, est néanmoins plus élevée par rapport aux autres bacheliers (18,5 ans pour les bacheliers technologiques et 18 ans pour les bacheliers L, ES et S).

Ceci peut représenter un avantage auprès des jurys d'admission recherchant une certaine maturité dans le choix de cette orientation en art.

Toutefois, ce fait est aussi révélateur d'une certaine fragilité scolaire, notamment parce que l'entrée dans l'enseignement professionnel reste encore, en France, une orientation par défaut dans nombre de spécialités.

La mention au baccalauréat constitue aussi un indicateur intéressant. En CPGE, la moitié des inscrits ont eu une mention bien ou très bien et neuf élèves sur dix ont accompli un parcours linéaire (Lemaire, 2008). Dans les classes préparatoires aux écoles supérieures d'art, $30 \%$ des bacheliers généraux ont obtenu la mention assez bien et près du quart les mentions bien et très bien. La moitié seulement a suivi une scolarité linéaire. Moins élitistes, ces classes comptent seulement trois bacheliers professionnels ayant eu une mention très bien ou bien parmi les dix-neuf.

\subsection{Une origine sociale modeste}

S’agissant des caractéristiques personnelles, ce sont essentiellement des hommes qui ont été admis avec un baccalauréat professionnel (douze hommes et sept femmes), alors que le taux de féminisation est important en classe préparatoire (64\%).

En outre, l'origine sociale pèse lourdement sur les bacheliers professionnels. Les dernières statistiques sur l'école (DEPP, 2014) rappellent que la voie professionnelle, désertée par les enfants de cadres (9\%), attire massivement les enfants d'ouvriers (41\%) qui, d'une manière générale, sortent moins souvent diplômés de l'enseignement secondaire (Vanhoffelen, 2010). Ces réalités sur l'orientation et la réussite se retrouvent au niveau de l'enseignement supérieur (Berthelot, 1987 ; Dubet, Martucelli, 1996).

Les arbitrages stratégiques à chaque étape décisive du cursus scolaire, selon sa situation sociale, (Boudon, 1973) amènent à apprécier différemment les risques, les coûts et les avantages d'un investissement dans une poursuite d'études (Landrier, Nakhili, 2010). De ce point de vue, l'orientation en classe préparatoire représente un risque élevé pour les milieux modestes. En effet, ce cursus ne garantit pas une réussite aux concours des écoles supérieures d'art.

Par ailleurs, bien que les frais de scolarité soient relativement peu élevés comparativement à l'offre privée ${ }^{5}$ l'année préparatoire reste indéniablement coûteuse, que ce soit en temps

5. Les frais d'inscription varient, selon les écoles, de 200 à $1100 €$ dans le secteur public et généralement 
ou en argent, d'autant plus qu'elle ouvre la voie sur des études longues, représentant ellesmêmes un investissement conséquent.

Cette poursuite d'études est donc plus facile à envisager pour ceux dont le projet est de rester en contact avec un environnement socioculturel dans lequel ils auraient trouvé des repères identificatoires. Dans ce rapport aux études, l'influence du milieu familial a son importance.

Tableau 2. Répartition par origine sociale des étudiants en 2013 (en \%)

\begin{tabular}{l|ccc}
\hline & $\begin{array}{c}\text { Classes préparatoires } \\
\text { aux écoles sup. d'art }\end{array}$ & $\begin{array}{c}\text { Étudiants en CPGE } \\
\text { en France }\end{array}$ & $\begin{array}{c}\text { Étudiants } \\
\text { en France }\end{array}$ \\
\hline Agriculteurs, artisans, commerçants & 13 & 11 & 10 \\
Cadres supérieurs & 31 & 50 & 30 \\
Professions intermédiaires & 19 & 12 & 12 \\
Employés & 8 & 10 & 12 \\
Ouvriers & 12 & 6 & 10 \\
Retraités, inactifs & 5 & 6 & 12 \\
Non renseignés & 12 & 5 & 14 \\
Total & 100 & 100 & 100 \\
\hline
\end{tabular}

Lecture : la part des enfants d'ouvriers inscrits en classe préparatoires publiques d'art est de $12 \%$.

CPGE : Classe préparatoire aux grandes écoles.

Sources : enquête IREDU, 2014 et DEPP, 2014 p. 195.

Des recherches conduites sur les CPGE soulignent, en effet, que les modalités d'accès ne reposent pas seulement sur le mérite scolaire du postulant. Une autre forme de sélection sociale se réalise par le capital culturel et des ressources économiques quapporte la famille (Bodin, 2007). Ainsi, les CPGE scolarisent $6 \%$ de bacheliers de père ouvrier et $50 \%$ de père cadre supérieur (DEPP, 2014).

En miroir, les classes préparatoires d'art présentent une composition sociale plus diversifiée, avec $12 \%$ d'enfants de père ouvrier et $31 \%$ de père cadre supérieur (tableau 2). En ne retenant que le statut le plus élevé de l'un des deux parents, tous baccalauréats confondus, $44 \%$ des inscrits ont des parents cadres supérieurs mais sept bacheliers professionnels sur dix-neuf se démarquent par une origine sociale plus modeste. De ce fait, ces élèves reconnaissent plus fréquemment rencontrer des difficultés financières : huit bacheliers professionnels précisent qu'elles sont nombreuses ou très sérieuses, ce qui ne concerne que $18 \%$ des autres répondants. De même, la proportion de bénéficiaires auparavant d'une bourse au lycée est plus importante (cinq bacheliers professionnels pour $18 \%$ des autres bacheliers).

au-dessus de $7500 €$ dans le secteur privé, indépendamment des frais de restauration et de logement. 
Au-delà de ce capital économique disponible assurant des conditions d'études favorables, le milieu familial apporte aussi des avantages socioculturels qui peuvent se convertir en bénéfices scolaires (Bourdieu, Passeron, 1964).

Le suivi sur plus de trente ans de l'évolution des pratiques culturelles des français montre que les parents de statut professionnel cadre supérieur ont des pratiques culturelles (lecture, bibliothèque, spectacle, musée ou exposition) plus développées que les autres milieux et exercent plus souvent une activité artistique en amateur. Or, ces pratiques familiales ont un impact certain sur les propres pratiques des enfants qui reproduisent les habitudes culturelles des parents (Donnat, 2011). La transmission d'un capital culturel familial ne s'exprime pas seulement dans les habitudes mais aussi dans les habitus, dispositions durablement forgées qui définissent le sens de l'orientation sociale. Ainsi, l'intérêt des héritiers se portera naturellement vers les savoirs savants plutôt que vers des savoirs accessibles par tous (Bourdieu, 1979).

La vocation artistique, elle-même, aurait du mal à s'exprimer si elle ne trouvait pas, d'une manière ou d'une autre, un soutien dans la famille, qu'il soit économique, social ou psychologique (Liot, 2004). La proportion des héritiers est loin d'être anecdotique dans les professions artistiques (Brenot, 1997 ; Coulangeon, 2004 ; Sorignet, 2006). La présence d'un parent artiste ayant servi de modèle identificatoire, un soutien de la famille dans un projet artistique (Sapiro, 2007) ou l'habitus et l' hexis corporelle ${ }^{6}$ que les enfants acquièrent dès leur plus jeune âge (Elias, 1986), expliquent bien des carrières et des talents (Blacking, 1980 ; Menger, 1997), tel un héritage familial à valoriser.

Dans notre population, quatre jeunes de l'enseignement professionnel ont un parent ayant suivi une formation artistique et dans deux situations, celui-ci exerce le métier d'artiste. Cette ouverture culturelle familiale s'exprime dans des proportions équivalentes aux autres bacheliers.

\subsection{Un choix moins affirmé}

L'appétence des élèves pour l'art en classe préparatoire est forte et s'inscrit dans le temps puisque la moitié des bacheliers non professionnels ont formulé ce choix avant le lycée. C'est moins souvent le cas des bacheliers professionnels, onze jeunes sur dix-sept répondants ont décidé de leur orientation vers des études artistiques à partir du lycée. Ils sont aussi moins nombreux à avoir bénéficié d'une spécialisation en art au baccalauréat : moins du tiers pour $70 \%$ des autres jeunes.

6. L'habitus désigne des dispositions générales (façon de faire et de réagir) résultant de l'intériorisation et de l'accumulation des apprentissages et savoirs inculqués par la famille, l'école, l'environnement social lors du processus de socialisation. L'hexis corporelle renvoie aux comportements physiques du corps (gestes, marche, posture..), variables d'une classe d'individus à une autre. 
De plus, le choix de la classe préparatoire n'est pas toujours l'objet d'un premier vœu, même si l'inclinaison allait plutôt vers des études artistiques (entrée directe en école supérieure d'art, faculté d'histoire de l'art, BTS design, MANAA...) : si onze bacheliers professionnels sur dix-huit répondants ont souhaité ,en première option, entrer en classe préparatoire, cela concerne les deux tiers des autres bacheliers.

Enfin, interrogés sur leur projet professionnel après six mois de cours, les bacheliers professionnels apparaissent plus hésitants. Contrairement à la tendance générale qui est d'espérer une admission en école supérieure d'art (85 \% des jeunes), ils envisagent moins cette possibilité (quatorze sur dix-neuf) et espèrent davantage une autre orientation (quatre élèves, dont deux en école d'architecture).

Leurs propos trahissent aussi une plus grande difficulté à se projeter dans l'avenir : treize bacheliers professionnels ne sont, en effet, pas en mesure de préciser un objectif de carrière. Cette situation d'incertitude est exprimée par moins de la moitié des autres bacheliers.

Au terme de cette première analyse, les bacheliers professionnels présentent un profil scolaire, un degré d'engagement et des origines socioculturelles qui laissent présager de plus grandes difficultés à entrer dans les savoirs savants valorisés en classe préparatoire.

Ces éléments de distinction n’ont cependant pas empêché leur candidature d'être retenue parmi les nombreuses présentées, notamment parce que les critères scolaires sont moins souvent pris en considération que l'approche artistique.

Dès lors, ces bacheliers atypiques peuvent avoir un positionnement tout aussi atypique au regard de la formation : justifient-ils leur choix par un discours plus vocationnel, sont-ils plus stratégiques par rapport à ces études ou expriment-ils davantage un désir d'appartenance sociale à un milieu ? Il convient ainsi de savoir ce qui différencie ces jeunes des autres bacheliers dans leur motivation à entreprendre des études artistiques et en quoi cela peut impacter leur adaptation à cette année préparatoire.

\section{Des « raisons d'étudier » comparables et un rapport aux études normalisé}

Les raisons qui les ont amenés à privilégier ce cursus méritent, en effet, d'être clarifiées puisqu'ils sont rares, statistiquement, à l'envisager.

Pour appréhender leurs motivations, cette recherche s'est appuyée sur les analyses de Dubet et Martucelli (1996) concernant les projets des lycéens. Les auteurs observent que les jeunes vivent leur expérience scolaire dans une recherche de cohérence entre le souci de vocation intellectuelle et d'accomplissement personnel dans les études, la prise de conscience des enjeux stratégiques du choix des études pour l'insertion, et le besoin de se forger une personnalité sociale répondant au désir d'appartenance à un groupe. 
Suivant cette approche théorique, trois questions ont été soumises aux élèves des classes préparatoires pour mieux cerner leurs motivations à étudier. Ces questions concernaient les raisons du choix d'une orientation en classe préparatoire $(\mathrm{Q} 1)$, les raisons du choix de la classe dans laquelle ils sont inscrits (Q2) et leurs objectifs pour cette année préparatoire (Q3). Les réponses qui leur étaient proposées étaient construites de façon à saisir l'importance des logiques d'action, supposées rationnelles par rapport aux valeurs (projet vocationnel), aux moyens (projet stratégique), ou au groupe social (projet de socialisation).

Sur ce dernier point, la classe préparatoire a comme singularité de se situer entre la fin d'un cursus secondaire et avant la reconnaissance du statut étudiant. De ce fait, la logique d'appartenance sociale est sans doute la plus difficile encore à définir. C'est pourquoi elle a été restreinte à l'adhésion recherchée par le bachelier auprès de l'entourage quant à son choix d'études.

\section{Graph 1- Les motivations des bacheliers pour les études artistiques}

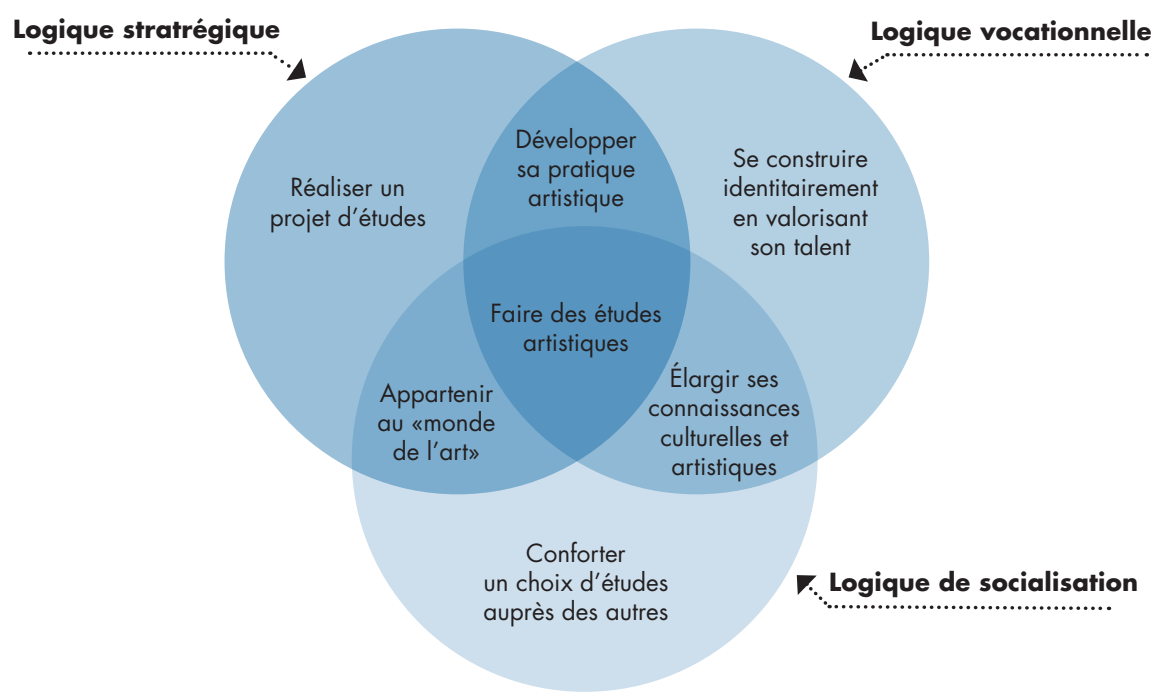

Lecture : Ce graphique illustre les raisons évoquées dans le questionnement selon les logiques des jeunes. Ainsi, «Élargir ses connaissances culturelles et artistiques » fait référence à deux logiques, vocationnelle et de socialisation.

Source : enquête IREDU, 2014.

Les précisions apportées par Paivandi (2011) ont permis de mieux spécifier ces différentes logiques d'action pour un public estudiantin. Néanmoins, elles sont apparues encore trop peu adaptées au contexte spécifique d'une formation adossée à un champ professionnel induisant une spécialisation des compétences en art. Le modèle de base a donc été enrichi par la mise en valeur des interactions entre les logiques d'action permettant de mieux saisir la dimension professionnalisante du parcours d'études artistiques (graphique 1). 
Dans cette seconde partie, les " raisons d'étudier " des bacheliers seront appréhendées au regard de ce modèle, d'abord d'un point de vue général, puis d'un point de vue plus spécifique en s'intéressant à celles retenues par les bacheliers professionnels. Par la suite, leurs attentes vis-à-vis des contenus enseignés et des conditions d'apprentissage seront identifiées.

\subsection{Une richesse des intentions}

La recherche d'une opportunité pour tirer profit de son potentiel est au carrefour d'enjeux stratégiques, identitaires et sociaux. Les bacheliers inscrits en classe préparatoire ont fait ce choix parce qu'il correspond à leur projet et que les conditions d'études étaient réunies pour les envisager (faire des études artistiques, L1, tableaux 3 et 3Bis).

Tableau 3. Spécification des logiques à partir des items des questions 1 (Q1), (Q2) et (Q3)

\begin{tabular}{|c|c|c|}
\hline \multirow{9}{*}{$\begin{array}{l}\text { Toutes logiques } \\
\text { confondues: } \\
\text { stratégique, } \\
\text { vocationnelle, sociale }\end{array}$} & \multicolumn{2}{|c|}{ L1 Faire des études artistiques } \\
\hline & Q1G Cela correspond à mon projet professionnel & $87 \%=$ \\
\hline & $\begin{array}{l}\text { Q2B La plaquette de présentation correspondait } \\
\text { à ce que je cherchais }\end{array}$ & $70 \%=$ \\
\hline & $\begin{array}{l}\text { Q2F Le contenu des études était en lien } \\
\text { avec mes compétences artistiques }\end{array}$ & $69 \%=$ \\
\hline & $\begin{array}{l}\text { Q2K Les conditions matérielles de l'école } \\
\text { étaient intéressantes }\end{array}$ & $70 \%+$ \\
\hline & $\begin{array}{l}\text { Q2D Les frais d'inscription et de scolarité } \\
\text { étaient accessibles }\end{array}$ & $84 \%=$ \\
\hline & Q2E C'était une école proche de ma famille, de mes amis & $41 \%=$ \\
\hline & Q2I C'était la seule classe préparatoire où j'ai été admis-e & $37 \%=$ \\
\hline & Q 1 H J'ai suivi le parcours d'un-e ami-e & $6 \%=$ \\
\hline
\end{tabular}

Tableau 3 Bis. Spécification des logiques à partir des items des questions 1 (Q1), (Q2) et (Q3)

\begin{tabular}{|c|c|c|c|c|c|c|}
\hline & \multicolumn{2}{|l|}{ Logique stratégique } & \multicolumn{2}{|c|}{ Logique vocationnelle } & \multicolumn{2}{|c|}{ Logique sociale } \\
\hline \multirow{7}{*}{$\begin{array}{l}\text { Logique } \\
\text { stratégique }\end{array}$} & \multicolumn{2}{|c|}{$\begin{array}{l}\text { L2 Réussir le concours } \\
\text { d'entrée aux ESA }\end{array}$} & \multicolumn{2}{|c|}{$\begin{array}{c}\text { L5 Développer } \\
\text { sa pratique artistique }\end{array}$} & \multicolumn{2}{|c|}{$\begin{array}{l}\text { L6 Appartenir aux } \\
\text { « mondes de l'art » }\end{array}$} \\
\hline & $\begin{array}{c}\text { Q1D C'est la voie la plus straté- } \\
\text { gique pour intégrer une école } \\
\text { supérieure d'art }\end{array}$ & $\begin{array}{l}90 \% \\
=\end{array}$ & \multirow{2}{*}{$\begin{array}{c}\text { Q1B J'ai de bons résul- } \\
\text { tats dans les activités } \\
\text { artistiques }\end{array}$} & \multirow{2}{*}{$\begin{array}{c}70 \% \\
+\end{array}$} & \multirow{2}{*}{$\begin{array}{l}\text { Q2H Cette école possé- } \\
\text { dait un large réseau de } \\
\text { professionnels et de } \\
\text { partenaires }\end{array}$} & \multirow{2}{*}{$\begin{array}{c}44 \% \\
=\end{array}$} \\
\hline & $\begin{array}{l}\text { Q2G Les taux de réussite aux } \\
\text { concours étaient élevés }\end{array}$ & $\begin{array}{c}68 \% \\
=\end{array}$ & & & & \\
\hline & $\begin{array}{l}\text { Q3A Un dossier personnel } \\
\text { artistique pour passer les } \\
\text { concours }\end{array}$ & $\begin{array}{l}76 \% \\
=\end{array}$ & \multirow{2}{*}{$\begin{array}{l}\text { Q2A Les temps proposés } \\
\text { de pratique artistique } \\
\text { étaient importants }\end{array}$} & \multirow{2}{*}{$\begin{array}{c}76 \% \\
=\end{array}$} & \multirow{2}{*}{$\begin{array}{l}\text { Q2C L'école était dans } \\
\text { une ville dynamique } \\
\text { sur le plan culturel et } \\
\text { artistique }\end{array}$} & \multirow{2}{*}{$\begin{array}{c}34 \% \\
+\end{array}$} \\
\hline & $\begin{array}{l}\text { Q3B Une préparation psycho- } \\
\text { logique au concours }\end{array}$ & $\begin{array}{c}33 \% \\
=\end{array}$ & & & & \\
\hline & $\begin{array}{l}\text { Q3C Une compréhension des } \\
\text { attentes d'un jury de concours }\end{array}$ & $\begin{array}{l}47 \% \\
= \\
\end{array}$ & \multirow{2}{*}{$\begin{array}{l}\text { Q3D Des techniques pour } \\
\text { développer mes compé- } \\
\text { tences artistiques }\end{array}$} & \multirow{2}{*}{$\begin{array}{c}73 \% \\
=\end{array}$} & \multirow{2}{*}{$\begin{array}{l}\text { Q3H Une connais- } \\
\text { sance des professions } \\
\text { artistiques }\end{array}$} & \multirow{2}{*}{$\begin{array}{c}28 \% \\
=\end{array}$} \\
\hline & $\begin{array}{l}\text { Q3I La capacité à défendre } \\
\text { oralement mes productions }\end{array}$ & $\begin{array}{l}63 \% \\
=\end{array}$ & & & & \\
\hline
\end{tabular}


(Suite tableau 3 Bis)

\begin{tabular}{|c|c|c|c|c|}
\hline \multirow{6}{*}{$\begin{array}{l}\text { Logique } \\
\text { vocationnelle }\end{array}$} & \multicolumn{2}{|c|}{$\begin{array}{c}\text { L3 Se définir } \\
\text { identitairement } \\
\text { en valorisant son talent }\end{array}$} & \multicolumn{2}{|c|}{$\begin{array}{l}\text { L7 Elargir ses connaissances } \\
\text { culturelles et artistiques }\end{array}$} \\
\hline & $\begin{array}{l}\text { Q1A Seules les activités } \\
\text { artistiques présentent un } \\
\text { intérêt pour moi }\end{array}$ & $\begin{array}{c}34 \% \\
-\end{array}$ & \multirow{2}{*}{$\begin{array}{l}\text { Q1J Avoir de meilleures } \\
\text { connaissances cultu- } \\
\text { relles et artistiques }\end{array}$} & \multirow[b]{2}{*}{$\begin{array}{c}96 \% \\
=\end{array}$} \\
\hline & $\begin{array}{l}\text { Q1F Faire des études } \\
\text { artistiques est une } \\
\text { évidence pour moi } \\
\text { depuis toujours }\end{array}$ & $\begin{array}{l}57 \% \\
=\end{array}$ & & \\
\hline & $\begin{array}{l}\text { Q1I C'est une opportu- } \\
\text { nité pour développer } \\
\text { mon potentiel artistique }\end{array}$ & $\begin{array}{c}96 \% \\
=\end{array}$ & \multirow{2}{*}{$\begin{array}{l}\text { Q3G Une compréhen- } \\
\text { sion des enjeux relatifs } \\
\text { à l'art }\end{array}$} & \multirow{2}{*}{$\begin{array}{l}41 \% \\
=\end{array}$} \\
\hline & $\begin{array}{l}\text { Q3E Une démarche de } \\
\text { réflexion autour de ma } \\
\text { pratique artistique }\end{array}$ & $\begin{array}{c}67 \% \\
=\end{array}$ & & \\
\hline & $\begin{array}{l}\text { Q3F La possibilité de } \\
\text { développer ma créativité } \\
\text { et mon originalité }\end{array}$ & $\begin{array}{c}72 \% \\
=\end{array}$ & $\begin{array}{l}\text { Q3J Une ouverture } \\
\text { culturelle sur la vie } \\
\text { artistique }\end{array}$ & $\begin{array}{c}56 \% \\
=\end{array}$ \\
\hline \multirow{6}{*}{$\begin{array}{l}\text { Logique } \\
\text { sociale }\end{array}$} & & & \multicolumn{2}{|c|}{$\begin{array}{l}\text { L4 Conforter auprès des autres } \\
\text { un choix d'études }\end{array}$} \\
\hline & & & $\begin{array}{l}\text { Q1C Un enseignant } \\
\text { m'a parlé de ce choix } \\
\text { d'études }\end{array}$ & $\begin{array}{c}27 \% \\
=\end{array}$ \\
\hline & & & $\begin{array}{l}\text { Q1E Mes parents } \\
\text { approuvent ce choix } \\
\text { d'études pour moi }\end{array}$ & $\begin{array}{c}78 \% \\
+\end{array}$ \\
\hline & & & $\begin{array}{l}\text { Q1K Je voulais mieux } \\
\text { définir mon orientation }\end{array}$ & $\begin{array}{c}69 \% \\
=\end{array}$ \\
\hline & & & $\begin{array}{l}\text { Q2J Ceux qui ont déjà } \\
\text { fait cette classe en } \\
\text { parlaient de façon } \\
\text { positive }\end{array}$ & $\begin{array}{l}71 \% \\
=\end{array}$ \\
\hline & & & $\begin{array}{l}\text { Q3K Des conseils pour } \\
\text { I'orientation }\end{array}$ & $\begin{array}{c}35 \% \\
=\end{array}$ \\
\hline
\end{tabular}

Note de lecture : Les signes + et - associés aux pourcentages indiquent une différence significative d'estimation des items proposés entre les bacheliers professionnels et les autres bacheliers, au seuil de 0,10 (test du khi²). Le signe positif correspond à un pourcentage plus élevé de bacheliers professionnels s'exprimant en faveur de l'item et inversement pour le signe négatif.

Lecture : $70 \%$ des bacheliers généraux sélectionnent l'item Q1B « J'ai de bons résultats dans les activités artistiques » à la question « Pourquoi avez-vous choisi de faire une classe préparatoire aux écoles supérieures d'art ? ». Les bacheliers professionnels sont significativement plus nombreux à choisir cet item pour expliquer leur choix (+).

Source : enquête IREDU, 2014. 
"Pouvoir entrer dans une école supérieure d'art" ou "avoir ses concours" sont des propos qui reviennent, tel un leitmotiv, dans les réponses des bacheliers lorsqu'il leur est demandé de définir leur réussite en classe préparatoire. La finalité de la classe préparatoire étant principalement d'augmenter les chances d'accès aux écoles supérieures d'art, la quasitotalité des élèves ont choisi cette orientation car elle leur semble être la voie la plus sûre pour y parvenir $(90 \%)$. La moitié des jeunes ont d'ailleurs déjà tenté une entrée directe en école d'art et mesurent donc le niveau d'exigence attendu aux concours. Les taux de réussite annoncés par les écoles ont influencé la décision d'un grand nombre $(68 \%)$.

Enfin, les trois quarts suivent cette scolarité pour renforcer le dossier artistique qu'ils devront soumettre aux jurys des concours et, pour $63 \%$, se préparer à le défendre oralement (réussir le concours d'entrée aux écoles supérieures d'art, L2).

De nombreux élèves conçoivent aussi ces études dans une logique de subjectivation sociale où il s'agit de se construire identitairement, indépendamment des rapports de production et de domination présents dans le champ des études. La classe préparatoire se présente ainsi comme une opportunité pour développer son potentiel artistique (pour $96 \%$ des élèves), sa créativité et son originalité (72\%), voire pour entrer dans une démarche de réflexion par rapport à sa pratique artistique $(67 \%)$. Plus de la moitié des bacheliers ( $57 \%$ ) se situent clairement dans ce registre, considérant que les études artistiques sont une évidence pour eux depuis toujours et, pour un tiers, que les activités artistiques représentent leur seul intérêt (se définir identitairement en valorisant son talent, L3).

Bénéficiant de l'approbation de leurs parents pour ce choix d'études (78 \%) et rassurés par un enseignant ( $27 \%)$ ou d'anciens élèves $(71 \%)$ sur l'intérêt de cette formation, les jeunes justifient le choix de la classe préparatoire par la nécessité de mieux préciser leur orientation (69 \%) et de bénéficier de conseils dans ce sens grâce aux intervenants de la formation, pour un tiers d'entre eux (conforter auprès des autres un choix d'études, L4).

Au-delà de ces logiques d'action qui caractérisent finalement, même à des degrés divers, tous les bacheliers qui poursuivent des études supérieures, les élèves font interagir ces logiques pour se construire un projet cohérent et porteur de sens. Un bachelier professionnel résume ainsi son année et cette appropriation des différentes rationalités qui ont justifié ce choix : "Mieux savoir où je veux aller et dans quel but, être pris dans une école d'art, avoir enrichi sa culture artistique culturelle et générale, avoir appris des techniques et à parler de son travail ».

$\mathrm{Au}$ carrefour des ambitions stratégiques et du besoin d'accomplissement personnel (développer sa pratique artistique, L5), nombreux sont les jeunes venus poser les fondements d'un projet professionnel (87\% des répondants). Celui-ci suppose un 
approfondissement de ses compétences à travers l'importance des temps consacrés à la pratique artistique (pour $76 \%$ ) et l'enseignement de techniques (73\%).

De même, le croisement des logiques stratégique et sociale (appartenir aux mondes de l'art, L6) amène les élèves les plus sensibles à la question de l'insertion professionnelle à envisager la classe préparatoire pour améliorer leur compréhension des « mondes de l'art» (Becker, 1975), par les réseaux socioprofessionnels de l'école (44\%), l'immersion dans la vie artistique de la ville $(34 \%)$ ou encore une meilleure connaissance des professions artistiques $(28 \%)$.

Enfin, les logiques sociale et vocationnelle des études (élargir ses connaissances culturelles et artistiques, L7) se concilient fort bien pour envisager la classe préparatoire comme l'opportunité d'élargir son horizon culturel et artistique, que ce soit explicitement par l'acquisition de nouvelles connaissances (pour $96 \%$ des élèves) ou indirectement par une plus grande ouverture culturelle sur la vie artistique (56\%) et une meilleure compréhension des enjeux relatifs à l'art (41\%).

\subsection{Des motifs d'étudier similaires}

Par rapport à cette situation d'ensemble, le positionnement des bacheliers professionnels a fait l'objet d'un regard particulier. Leur profil spécifique et leur socialisation secondaire orientée vers des enjeux de production devraient théoriquement les amener à privilégier des logiques d'action pragmatiques plutôt que des logiques conceptuelles. En effet, le risque pris à entrer en classe préparatoire pour des bacheliers professionnels pourrait justifier le besoin d'être rassurés sur le choix d'études (L4). De même, leur éloignement d'une culture élitiste serait de nature à les inciter majoritairement à mieux se situer dans le champ professionnel artistique (L6).

Une autre hypothèse considère que leur cursus est souvent marqué par des difficultés scolaires. Il ne serait donc pas surprenant de les trouver autour d'une logique stratégique de survie, définie par un objectif à court terme de réussite aux concours (L2). Enfin, compte tenu des candidatures nombreuses à l'entrée en classe préparatoire et des modalités particulières de sélection, ces bacheliers atypiques pourraient s'être distingués par leur potentiel et leur intérêt artistiques (L5), dans un contexte de scolarisation adapté à leur projet (L1). En revanche, il est moins attendu, au regard de leur parcours scolaire et de leur socialisation antérieure, de les retrouver dans un rapport hédoniste à la culture et aux connaissances artistiques (L7), ou dans une réflexion identitaire autour de leur potentiel de création et d'innovation (L3).

Les analyses de discours ne confirment pas ces projections relatives à une mobilisation accrue des bacheliers professionnels sur certaines logiques d'action et leur évasion dans d'autres. Encouragés à s'exprimer librement sur la question " pour vous, réussir votre année en classe préparatoire, c’est... ", leurs propos ressemblent à ceux des autres 
jeunes. Six bacheliers professionnels énoncent des réponses purement stratégiques : "Obtenir les concours", "Pouvoir entrer dans une école supérieure de beaux-arts facilement ", "Pouvoir poursuivre mes études dans le monde de l'art".

Deux bacheliers illustrent un rapport vocationnel aux études : "Réussir à avoir une réflexion sur son travail et les différents types d'art qui m'entourent ainsi qu'une meilleure expression graphique"; "Développer ma pratique plastique, rencontrer des artistes et des professionnels". Les autres s'expriment de manière plus large et entrecroisent les réponses de types vocationnel, stratégique et social. Ainsi les verbes "avoir", "développer ", "découvrir ", "gagner " sont conjugués de diverses façons : "Avoir assimilé plusieurs points de vue et se différencier artistiquement des autres et réussir les concours souhaités", "Découvrir, apprendre, s'ouvrir et travailler", "Développer ma pratique plastique, rencontrer des artistes et des professionnels", "Obtenir une école et développer mes connaissances en art".

La taille de l'échantillon rend les analyses statistiques fragiles, avec un écart significatif observé seulement sur les items qui introduisent un rapport réaliste aux études (tableaux 3 et 3 bis).

Les bacheliers professionnels expriment plus souvent le sentiment d'être performants dans les activités artistiques et d'avoir bénéficié de l'adhésion de leur famille à leur projet d'études.

Ceci peut être interprété comme l'intériorisation de difficultés rencontrées dans le supérieur qui les amène à s'engager dans ces formations artistiques seulement après s'être assurés d'en avoir le potentiel, de leur point de vue et du point de vue plus objectif de leurs proches.

Pour justifier leur choix, ils ont accordé aussi plus d'importance aux conditions matérielles offertes par l'école et au dynamisme culturel et artistique de la ville d'accueil. Leur plus forte mobilité géographique, comparativement aux autres jeunes, pourrait expliquer cet intérêt sur ces deux items?

\subsection{Des attentes conformes}

Si les motivations des bacheliers professionnels ne diffèrent pas tant de celles des autres jeunes, leur rapport aux études ne déroge pas non plus à la tendance générale.

Les élèves sont nombreux à exprimer un intérêt fort pour les enseignements appliqués, cours pratiques (dessin, couleur, peinture, volume, photo, sculpture...) (79\%) et les workshops, ateliers de création et d'expérimentation (63\%). Les rencontres avec des professionnels (artistes, critiques, historiens de l'art...) et le suivi personnalisé dans

7. La densité du réseau des écoles publiques permet à $18 \%$ des admis d'être inscrits dans l'école de la ville où ils ont passé leur baccalauréat. Cette situation ne concerne que deux bacheliers professionnels sur dix-neuf. 
l'élaboration et la rédaction du dossier artistique mobilisent, dans des proportions équivalentes, l'intérêt prononcé de la majorité des jeunes (59\%).

Dans une moindre mesure, ce qui a trait aux concours motive moins de bacheliers, la préparation et les jurys blancs n'intéressant fortement que la moitié des jeunes, tout comme les sessions de présentation et d'argumentation orale autour du dossier artistique.

Enfin, les élèves sont minoritaires à manifester un engouement pour les temps de formation en lien avec les cours théoriques (culture générale, anglais, histoire de l'art... $47 \%)$ ou les conférences, les visites de musées et les expositions (43\%).

Les bacheliers professionnels formulent les mêmes attentes que les autres bacheliers. Les améliorations souhaitées iraient prioritairement vers plus de suivi individualisé autour du dossier artistique personnel, d'enseignements en lien avec les techniques et d'occasions de débattre avec des artistes (pour plus de $40 \%$ ). De même, plus du quart des élèves plaident pour un renforcement des activités sollicitant un travail personnel de production artistique et d'ouverture sur les acteurs du monde de l'art et de la création et un sur cinq aimerait avoir plus d'occasions d'être évalué sur sa pratique artistique.

Viennent ensuite les demandes en faveur d'une meilleure préparation aux concours, avec plus d'enseignements orientés vers cette finalité, d'examens blancs pour s'autoévaluer par rapport aux exigences du concours, d'encouragements de la part des enseignants et de contenus théoriques ou de connaissances en art (environ $20 \%$ ).

Ainsi, rien ne distingue radicalement les bacheliers professionnels des autres jeunes pour leurs motivations et leur rapport aux enseignements.

Leur comportement scolaire quant à la perception du temps d'études diffère cependant. Bien que les différences soient non significatives en raison des effectifs considérés et de l'importance des variations autour de l'estimation moyenne, les bacheliers professionnels semblent consacrer moins d'heures de suivi des enseignements (33 heures pour 34,5 heures déclarées par les bacheliers L, ES et S) et au travail personnel en dehors des cours (10 heures pour 13 heures déclarées par les bacheliers généraux).

En temps cumulés, ils accordent en moyenne 43 heures à leur formation, soit près de 4,5 heures de moins que les bacheliers L, ES et $S$ majoritairement représentés dans cette formation (tableau 4) ${ }^{8}$. Il est possible que ce moindre investissement soit le reflet de leur engagement plus modéré dans ces études ou d'habitudes scolaires acquises dans le secondaire. La proportion de bacheliers professionnels qui exprime des difficultés d'adaptation à la formation n'est toutefois pas très différente de celle des autres bache-

8. À titre de comparaison, les étudiants en STS déclarent en moyenne 30 heures d'enseignements et 10 heures de travail personnel, les étudiants de CPGE indiquent respectivement 33 et 24 heures (OVE, 2011). 
liers, qu'il s'agisse du niveau de connaissances artistiques et culturelles (29\%), du rythme de travail (42\%) ou du climat scolaire et de l'esprit de compétition (19\%). Dans l'ensemble, ils sont plutôt satisfaits de l'ambiance, comme l'exprime ce bachelier professionnel : "pas d'esprit de compétition ici... au contraire, un soutien et un esprit de communauté."

Tableau 4 . Temps moyen de travail par semaine selon l'origine scolaire des élèves

\begin{tabular}{l|c|ccc}
\hline \multicolumn{1}{c|}{ Profil des bacheliers } & $\begin{array}{c}\text { Effectifs } \\
\text { répondants }\end{array}$ & à la formation & $\begin{array}{c}\text { Heures consacrées } \\
\text { au travail personnel }\end{array}$ & Total \\
\hline Bac Professionnel & 16 & $33(6,4)$ & $10(5,9)$ & $43(9,6)$ \\
Bac Technologique & 26 & $33(7,1)$ & $11(7,8)$ & $44(12,4)$ \\
Bac L, S, ES & 254 & $34,5(5,6)$ & $13(7,2)$ & $47,5(8,9)$ \\
Total & 296 & $34(5,8)$ & $12(7,1)$ & $46(9,3)$ \\
\hline
\end{tabular}

Lecture : En moyenne, les 16 bacheliers professionnels répondants déclarent suivre 33 heures de cours par semaine et travailler en dehors des cours 10 heures par semaine, soit un total de 43 heures consacrées à la formation (entre parenthèses sont indiqués les écart-types).

Bac L : littéraire ; S : scientifique ; ES : économique et social.

Source : enquête IREDU, 2014.

C'est sans doute dans ce climat positif, comparable à celui évoqué par Buisson-Fenet et Landrier (2008) à propos d'une classe préparatoire accueillant des élèves situés en zone d'éducation prioritaire, qu'il faut trouver les explications d'une normalisation du rapport aux études des inscrits en classe préparatoire aux écoles supérieures d'art.

Bien que l'objectif du concours reste une priorité donnée à cette année, l'esprit de compétition ne semble pas de mise. L'enjeu de cette année est d' "avoir développé notre sensibilité, d'apprendre à connaître nos points forts et nos points faibles et d'avoir pu définir notre orientation. Cette année nous permet de nous exprimer ". Cette préoccupation liée à la connaissance de soi et à l'orientation est assumée par les responsables des structures, la moitié en faisant même une priorité de la formation. Comme l'explicite une directrice, "les classes préparatoires aident les jeunes à choisir une orientation appropriée à leurs aptitudes et à leur souhait professionnel en les confrontant à la culture et à l'art, en leur permettant, tout au long de cette année, d'évaluer leur motivation, leur curiosité et leurs capacités".

Plus subtilement, l'intention des responsables est de les éclairer à travers cette approche sur ce qui se joue dans les écoles supérieures d'art pour réussir un cursus complet, de sorte à pouvoir "se passionner pour la pratique artistique, tout en sortant du " fantasme de l'artiste", pour reprendre les propos d'un directeur.

De plus, l'organisation pédagogique de cette année se rapproche des pratiques véhiculées dans l'enseignement secondaire professionnel, à savoir une importance accordée à la pratique (65\% du temps de formation, avec plus ou moins de variation selon les écoles) et à l'acquisition de compétences en lien avec l'exercice professionnel. 
Pour ces bacheliers, incités plus souvent à s'inscrire dans une démarche de cheminement en raison du rapport particulier aux études que leur milieu et leur scolarisation antérieure ont instauré, cette proximité avec la réalité professionnelle contribue à donner du sens aux apprentissages (Bautier, Rochex, 1998).

Ce climat d'étude qui encourage moins à se distinguer des autres qu'à se découvrir soimême, place les bacheliers, quels que soient leurs cursus, sur un même pied d'égalité dans le sens où les connaissances acquises et les compétences scolaires ne déterminent pas nécessairement la qualité du processus de maturation individuelle.

Ainsi, le moindre capital socioculturel qui caractérise les bacheliers professionnels, eu égard à leurs origines modestes et à leur formation secondaire, ne leur donne pas le sentiment d'être plus confrontés que les autres élèves à des études ardues et ne prédétermine en rien leur intérêt pour les enseignements dispensés.

\section{Conclusion}

Cet article se présente comme une contribution supplémentaire pour une meilleure connaissance des déterminants de l'orientation des bacheliers professionnels qui s'aventurent vers des formations élitistes, particulièrement risquées pour eux.

Première étape d'un cursus long rythmé par des temps forts de sélection académique, la réussite aux concours n'est cependant qu'une mission parmi d'autres, toutes aussi essentielles, attribuée à la classe préparatoire publique aux écoles supérieures d'art par les responsables et les élèves. Cette année est aussi vécue comme un temps de formation artistique et joue, par ailleurs, un rôle propédeutique pour les jeunes en phase exploratoire ou de construction de projet. Pour cette année davantage centrée sur le développement personnel que sur un rapport concurrentiel entre élèves, le profil social et scolaire des bacheliers professionnels ne semble plus être un élément de distinction.

Plus précisément, cette recherche montre que leur profil atypique n’a pas pesé sur les logiques qui ont justifié ce choix d'études, pas plus que sur l'intérêt porté aux enseignements.

Peu visibles au niveau de chaque école où ils ne sont parfois que l'unique représentant de leur formation secondaire, ces bacheliers professionnels passent donc inaperçus au regard du rapport qu'ils entretiennent avec les savoirs et les enseignements. Se fondant dans un discours partagé par tous et s'instituant comme une norme, la classe préparatoire exerce alors un effet lissant sur leurs origines scolaire et sociale. En d'autres termes, elle les dépossède des stigmates généralement attribués aux bacheliers professionnels qui investissent des études supérieures longues.

Face à un public qui paraît composite, se dessine l'image d'une population homogène dans la justification de ses choix et l'intérêt porté aux enseignements. La classe préparatoire aux écoles supérieures d'art est une année privilégiée, un moment clé donnant à l'élève la possibilité de "s’épanouir dans ses passions, ses projets, trouver sa voie et s'investir » ou, comme 
le formule plus simplement ce bachelier professionnel, "être heureux et m’épanouir dans mon existence ". Univers particulier et singulier dans ce rapport hédonique aux études, le monde de l'art sait passer sous silence les différences individuelles pour rassembler, dans un même propos, des élèves distincts socialement et scolairement.

Pour appréhender leur rapport aux études, cette recherche s'appuie sur un modèle présentant les logiques d'action et leurs interactions, plus à même de refléter une spécialisation des compétences propres aux filières adossées à un champ professionnel. Illustration originale de ces situations généralement peu observées, l'analyse a toutefois porté sur un échantillon limité de bacheliers professionnels et sur un contexte de scolarisation spécifique. Ce modèle doit encore être éprouvé pour déterminer dans quelle mesure il peut être transposé à d'autres formations vocationnelles comme la santé, l'enseignement ou le sport. En s'inscrivant dans cette démarche, les conclusions de cette recherche sur les bacheliers professionnels engagés dans les études supérieures pourront être éventuellement généralisées.

À ce jour, les conclusions de cette recherche invitent à modifier la lecture en négatif faite sur les bacheliers professionnels qui se trouvent engagés dans des études longues, sélectives et aux contenus d'enseignement élitistes. Par leur engagement artistique, leurs difficultés à se projeter dans le temps ou leur implication dans le travail personnel, ces élèves ne présentent pas les garanties d'une réussite aisée. Ils ont d'ailleurs moins d'une année pour exprimer plus concrètement leur détermination à investir le champ artistique. Cependant, comme ils ont volontairement choisi cette orientation et emprunté les habits de la conformité, le rapport aux études de ces bacheliers professionnels est vécu comme positif et constructif.

Ainsi, les classes préparatoires ouvertes sur une spécialisation des compétences au regard d'un champ professionnel peuvent représenter des alternatives heureuses de poursuite d'études dans le supérieur.

\section{Bibliographie}

Bautier E., Rochex J.-Y. (1998), L'expérience scolaire des "nouveaux lycéens": démocratisation ou massification ?, Paris, Armand Colin.

Becker G. (1975), « Le capital humain et la répartition des revenus : un approche analytique ", extrait du Capital humain de Becker, traduit en français, 11 p.

Beaud S. (2002), $80 \%$ au bac, et après? Les enfants de la démocratisation scolaire, Paris, La Découverte.

Beaud S., Pialoux M. (2001), "Les bacs pro à l'université. Récit d'une impasse ", Revue française de pédagogie, 136, pp. 87-95. 
Berthelot J.-M (1987), " De la terminale aux études post-bac [Itinéraire et logiques d'orientation] ", Revue française de pédagogie, 81, pp. 5-15.

Blacking J. (1980), Le sens musical, Paris, Éditions de Minuit.

Bodin Y. (2007), Diversité sociale dans les classes préparatoires aux grandes écoles : mettre fin à une forme de "délit d'initié", Rapport d'information n 41, au nom de la Commission des affaires culturelles et de la mission d'information, déposé le 12 septembre 2007 http://www.senat.fr/rap/r06-441/r06-441.html

Boudon R. (1973), L'inégalité des chances, Paris, Armand Colin.

Bourdieu P. (1979), La distinction, critique sociale du jugement, Paris, Éditions de Minuit.

Bourdieu P., Passeron J.-C. (1964), Les héritiers. Les étudiants et la culture, Paris, Éditions de Minuit.

Brenot P. (1997), Le génie et la folie, en peinture, musique et littérature, Paris, Odile Jacob.

Buisson-Fenet H., Landrier S. (2008), «Être ou pas ? Discrimination positive et révélation du rapport au savoir. Le cas d'une " prépa ZEP » de province ", Éducation et Sociétés 2008/1, n' 21, pp. 67-80.

Coulon A. (1997), Le métier d'étudiant. L'entrée dans la vie universitaire, Paris, PUF.

Coulangeon P. (2004), Les musiciens interprètes en France, Paris, La Documentation française.

Danner M., Guégnard C., Albandea I. (2014), Motivation et poursuite de parcours des étudiants en Ecole supérieure d'art ayant suivi une formation dans une école préparatoire publique ou privée, Rapport pour le ministère de la Culture et de la Communication, IREDU-CEREQ, université de Bourgogne.

DEPP (2014), Repères et références statistiques, ministère de l'Éducation nationale.

Donnat O. (2011), "Pratiques culturelles, 1973-2008. Dynamiques générationnelles et pesanteurs sociales ", Culture Etudes, n 7 , DEPS, ministère de la Culture et de la Communication.

Dubet F., Martucelli D. (1996), À l'école. Sociologie de l'expérience scolaire, Paris, Édition du Seuil.

Elias N. (éd. orig. 1986, 1991), Mozart, sociologie d’un génie, Paris, Édition du Seuil.

Felouzis G. (2001), La condition étudiante, Paris, PUF.

Fouquet S. (2014), "Parcours et réussite en licence et en master à l'université ", Note d'information, $\mathrm{n}^{\circ}$ 10-06, ministère de l'Enseignement supérieur et Recherche.

Frank R. H., Cook P. J. (1995), The Winner-Take-All Society, The Free Press, New York. 
Freidson E. (1994), "Pourquoi l'art ne peut pas être une profession ", in L'art de la recherche, Essais en l'honneur de Raymonde Moulin, pp. 117-136, Paris, La Documentation française.

Landrier S., Nakhili N. (2010), «Comment l'orientation contribue aux inégalités de parcours scolaires en France ", Formation Emploi, n 109, pp. 23-36.

Lemaire S. (2008), « Disparités d'accès et parcours en classes préparatoires ", Note d'information, $\mathrm{n}^{\circ} 12.04$, DEPP, ministère de l'Éducation nationale, ministère de l'Enseignement supérieur et de la Recherche.

Liot F. (2004), Le métier d'artiste, Paris, L'Harmattan.

Menger P.-M. (1997), La profession de comédien, Département des études et de la prospective, Paris, ministère de la Culture et de la Communication.

Menger P.-M. (2002), Portrait de l'artiste en travailleur. Métamorphoses du capitalisme, Paris, Seuil, «La République des idées".

Ministère de l'Éducation nationale, de l'Enseignement supérieur et de la Recherche (2015), L'état de l'enseignement supérieur et de la recherche en France, 49 indicateurs.

OVE (2011), Enquête Conditions de vie des étudiants 2010, La Documentation française.

Paivandi S. (2011) «La professionnalisation de l'Université française : la perspective étudiante ", Cahiers de la recherche sur l'éducation et les savoirs, hors-série n³ 3, pp. 167-186.

Prélot P.H. (1992), "Les établissements privés d'enseignement artistique ", Savoir Education Formation, 1, pp. 33-43.

Sapiro G. (2007), "La vocation artistique entre don et don de soi ", Actes de la recherche en sciences sociales, 168 , pp. 4-1

Sorignet P.-E. (2006), "Danser au-delà de la douleur ", Actes de la recherche en sciences sociales 163 , pp. 40-61.

Sulzer E. (2000), "Les stratégies d'orientation face aux transformations de l'offre de formation ", Formation Emploi, n 69, pp. 21-36.

Talbot P. (1996), "Les écoles d'Art : du libre usage du propre... ", in Pourquoi étudier?, Agora débats/jeunesse, pp. 31-40.

Vanhoffelen A. (2010), "Les bacheliers du panel 1995 : évolution et analyse des parcours ", Note d'information, $\mathrm{n}^{\circ} 10.13$, DEPP, ministère de l'Éducation nationale. 